\title{
Effect of assortative mating on genetic gain and inbreeding in aquaculture selective breeding programs
}

\author{
M. Saura, \\ B.Villanueva, J.Fe r n á n d e z, M. A. Tor o
}

\section{Keywords:}

Assortative mating

Aquaculture

Between-family variance

Common environmental effect

Genetic gain

Inbreeding

\begin{abstract}
A B S T R A C T
In this simulation study, the effect of the mating scheme on genetic gain and inbreeding has been explored for aquaculture selection programs where tank effects and large family sizes are common. Different selection methods were investigated (individual, family, sib, combined and within-family selection). Our results suggest that under family and sib selection, genetic gain was increased with assortative mating in comparison to random mating. The advantage of assortative mating increased when common environmental effects were present. Contrarily, a decrease in genetic gain was observed with disassortative mating, except for the case of within-family selection. The advantage of assortative mating over random mating was due to the increase in the betweenfamily component of the additive genetic variance that was exacerbated with the presence of common environmental effects. Under family and sib selection, the join effect of assortative mating and common environmental effects produced an increase in genetic gain of around 80 and $40 \%$ at early generations, and around 10 and $60 \%$ at later generations, respectively. Inbreeding was low under family selection for all mating schemes but much higher under sib selection when assortative mating was performed. In fact, the inbreeding coefficient after 10 generations of selection was $300 \%$ higher when assortative matings were performed under sib selection, compared to random matings. This was due to the fact that under sib selection, matings were based on family means, leading to an increased frequency of within-family matings. To our knowledge, this is the first study that investigates the effect of the mating scheme on genetic gain and inbreeding in an aquaculture context where family sizes are large and tank effects are present, and shows that assortative mating can substantially enhance the response to selection, particularly when family selection methods are applied.

Statement of relevance: Our article complies with the Policy Statement for submission of manuscripts to the Genetics Section, as it provides insight into the issue of breeding programs. Here, we have connected previous work in the field to address new questions, focusing on how the mating scheme may affect both genetic gain and inbreeding in aquaculture selection programs, where family sizes are typically large and tank effects are usually present.

In fish species, it is possible to consider different mating schemes because fecundity is high and because in vitro fertilization is often possible. A particular problem in aquaculture breeding programs is the impossibility of tagging physically newborn individuals. Given this, a common practice in aquaculture is to rear families in separate tanks until the fish are large enough to be individually tagged. This introduces an environmental effect common to the members of the same family (tank effect) which can lead to a reduction of the response to selection that needs to be considered.

We studied here the efficiency of different selection methods in terms of genetic gain and inbreeding and investigated the effect of the mating scheme to optimize breeding programs in aquaculture when tank effects are present. We have shown that assortative mating can substantially enhance the response to selection, particularly when family selection methods are applied and tank effects are present. To our knowledge, the effect of the mating scheme in an aquaculture context has never been addressed before. Our results suggest that assortative mating in the presence of common environmental variance may be considered in selection programs in aquaculture. Our conclusions will help breeders make optimal mating choices.
\end{abstract}

\section{Introduction}

The potential for high genetic gains through selective breeding are well documented for aquatic species, particularly due to the possibility 
of applying high selection pressures as a consequence of their high reproductive capacity (see Gjedrem and Baranski, 2009 for a review). Applying high selection pressures, however, can lead to high increases in inbreeding and, consequently, to reductions in fitness (i.e., inbreeding depression), genetic variance and long-term genetic progress.

The levels of genetic gain and inbreeding achieved in a selection program are highly dependent on the selection decisions taken. Traditionally, the most relevant selection methods for aquatic species have been individual and family selection (Gjedrem and Baranski, 2009). Individual selection is based on individual phenotypic values and gives the same weight to both between-family and within-family components. This method is easy to apply and has been for many years the most commonly used method of selection in aquaculture, particularly for improving growth (Neira et al., 2006; Gjedrem, 2012). With family selection, individuals are selected based on their family mean, i.e. all weight is given to the family component.

Although the genetic gain achieved depends mostly on selection decisions, the mating design may also affect the selection response. Theoretical studies (Falconer and Mackay, 1996; Lynch and Walsh, 1998) have demonstrated that, in the absence of selection, assortative matings (i.e., matings between individuals of similar phenotype) can increase the additive genetic variance of a quantitative trait. In particular, under the infinitesimal model assortative matings increase the between-family variance although does not affect the within-family variance provided the number of loci controlling the trait is not small (Falconer and Mackay 1996). Breese (1956) and James and McBride (1958) were the first to suggest that assortative matings could be used to increase the response to selection and this increase has been demonstrated empirically in a number of studies (e.g. experiments with Drosophila, by McBride and Robertson, 1963).

A particular problem in aquaculture breeding programs is the impossibility of tagging physically newborn individuals. Given this, a common practice in aquaculture is to rear families in separate tanks until the fish are large enough to be individually tagged. This introduces an environmental effect common to the members of the same family (usually known as the tank effect) which can lead to a reduction of the response to selection (Gjedrem and Baranski, 2009).

The effect of assortative matings on genetic gain and inbreeding has not been investigated in the aquaculture context, where most species display external fertilization (and therefore matings can be directed), family sizes are large and tank effects are usually present. The objective of this study was therefore to examine, through computer simulations, this effect under different selection methods applied in aquaculture breeding programs.

\section{Methods}

\subsection{Simulation of the population}

One hundred founder individuals ( 50 sires and 50 dams) were generated. The genome was composed of ten chromosomes of one Morgan each. The trait under selection was assumed to be controlled by 100 biallelic additive QTLs per chromosome. QTLs were evenly distributed across the chromosomes. Linkage and Hardy-Weinberg equilibrium were assumed. The genotypic values for each QTL were $a, 0$ and $-a$ for individuals with genotype $A A, A a$ and $a a$, respectively. Alleles were chosen at random with probability $p=0.5$. Phenotypic variance was set to one so that the additive genetic variance was equal to the heritability. Two heritabilities $\left(h^{2}=0.1\right.$ and 0.4$)$ were simulated. The $a$ value was calculated such that $2 p(1-p) a^{2}=h^{2} / 1000$. The between and within components of the additive genetic variance were calculated through standard ANOVA on the additive genetic values.

Generation $0(t=0)$ consisted of unselected individuals with family structure and was obtained from mating at random the founder individuals. It was composed by 50 families of 100 full-sibs (half of each sex) for all scenarios but sib selection, where (50) families were composed by 200 full-sibs (half of each sex), as explained below. The population was subjected to artificial selection during ten discrete generations. At $t \geq 0$, the genotype for each individual was obtained by sampling at random one gamete from each parent. The probability of recombination between adjacent QTLs was 0.01, according to the length of each chromosome. Common environmental (tank) effect was sampled from a normal $N\left(0, c^{2}\right)$, where $c^{2}$ is the proportion of phenotypic variance due to common environmental effects. Two values for $c^{2}(0.0$ and 0.4 ) were considered. Phenotypic values were obtained by adding a normally distributed individual environmental effect with mean zero and variance $V_{E}$ to the sum of genetic value and common environmental effect.

The percentage of individuals selected at each generation was $2 \%$ in all scenarios; i.e. 100 individuals ( 50 males and 50 females) were always used as breeders to produce the next generation that was composed by 50 families, as indicated above. One hundred replicates were run for all scenarios and the results presented are averages over replicates.

\subsection{Selection methods}

\section{Different selection methods were investigated including:}

(i) Random selection. The 50 males and 50 females were selected at random from the 50 families.

(ii) Individual selection. The 50 males and 50 females with the highest phenotypic values were selected.

(iii) Family selection. First, a number of families (1 or 25) with the highest average phenotypic value were selected. Then, 50 or 2 individuals of each sex were selected at random from these selected families.

(iv) Sib selection. This is a particular case of family selection that is widely used in aquaculture for improving traits that cannot be measured on live individuals (e.g. disease resistance or fillet quality traits), and consists of selecting candidates based on phenotypic records obtained on their sibs. Under sib selection, the number of offspring simulated per family was 200 . One hundred of them were measured for the quantitative trait and the remaining 100 sibs were the selection candidates. As with family selection, 1 or 25 families with the highest average phenotypic value were first selected. Then, 50 or 2 individuals of each sex were selected at random from these selected families.

(v) Combined selection. The weights given to the family mean and the within-family deviations were optimized for maximizing the accuracy of the index (Falconer and Mackay 1996). Then, the 50 males and 50 females with the highest index values were selected.

For completeness, we also investigated an additional selection scenario:

(vi) Within-family selection. One male and one female (those with the highest phenotypic values) within each of the 50 families were selected.

\subsection{Mating schemes}

Three alternative mating schemes were evaluated: RM (random mating of selected parents), AM (assortative matings) and DM (disassortative matings or matings between individuals of dissimilar phenotypes). In individual, family and within-family selection AM and DM were based on individual phenotypic values while in sib selection they were based on the family means (in practice, individual phenotypes would be unavailable). In combined selection schemes, AM and DM were based on index values. 


\section{Results}

\subsection{Random mating}

Table 1 shows the cumulative genetic gain and inbreeding observed at generations 1,5 , and 10 with RM after selecting individuals using different methods, for different values of $h^{2}$ and $c^{2}$. Results were in agreement with theory (Falconer and Mackay 1996). Genetic gain was always higher in the absence of common environmental effects and when $h^{2}$ is high (0.4). In general, combined selection was the method giving the highest genetic gain, followed by individual selection when $h^{2}=$ $c^{2}=0.4$. In contrast, for $h^{2}=0.1$ and $c^{2}=0.0$, strong family (sib) selection (i.e. selecting a single family) gave higher genetic gain than individual selection in early generations. This is expected, as optimal conditions for family selection are low $h^{2}$ and $c^{2}$ and large family sizes (Falconer and Mackay 1996). The latest could also be the reason for having a minimal advantage of individual selection over family ( $\mathrm{sib}$ ) selection in early generations $(t \leq 5)$ with the highest $h^{2}$ (and $c^{2}=0.0$ ). Genetic gain under family (sib) selection decreased drastically when decreasing the intensity of selection (i.e. selecting 25 families) and was always lower than that obtained with individual selection. When comparing within-family selection with individual selection, the former could be preferable in the long term $(t=10)$ when common environmental effects are present (i.e. $c^{2}=0.4$ ), as genetic gain was very similar with both selection methods but inbreeding was much lower with withinfamily selection ( 0.03 versus 0.38 at $t=10)$. In any case, family and sib selection were the methods producing the highest levels of inbreeding when a single family was selected.

The effect of selection on the additive genetic variance was also in agreement with theory. Fig. 1 shows the additive genetic variance across generations under family and sib selection when 25/50 families were selected (the selection methods where the effect of non-random mating was more evident). Under RM, both selection methods gave similar results. The highest decrease in the additive genetic variance occurred in the first generation of selection (Bulmer effect, Bulmer, 1971). With $c^{2}=0.4$ the additive genetic variance across generations was higher than with $c^{2}=0.0$, which is due to the between-family component, but not to the within family component of the variance, that is only affected by the inbreeding.

\subsection{Non-random mating}

All the results presented here refer to the effect of AM (and DM) when compared to RM. The only scenarios where the mating system had a noticeable effect were those under family and sib selection
(Table 2). The effect of the mating system was particularly strong when 25 out of 50 families were selected and $c^{2}=0.4$, particularly in later generations. For $c^{2}=0.4$ and $h^{2}=0.1$, AM led to an increase in genetic gain of around 80 and $40 \%$ at $t=1$, and around 10 and $60 \%$ at $5 \leq t \leq 10$, under family and sib selection, respectively, when compared to RM. For $h^{2}=c^{2}=0.4$ the advantage of AM increased over generations (from about 10 to $40 \%$ under both selection methods, Table 2). On the other hand, in general DM led to a decrease in genetic gain across generations due to the decrease in the between-family genetic variance, especially for $h^{2}=0.4$ (Figs. 2 and 3). The only method under which DM produced an increase of genetic gain was within-family selection and this was due to an increase in the within-family component of the additive genetic variance (data not shown).

The large increases in genetic gain with AM under sib selection were accompanied by much larger increases in inbreeding (inbreeding is only presented for the scenarios where non-random mating was different from RM, i.e. 25/50, Table 2). In fact, inbreeding was up to $300 \%$ higher with AM than with RM $\left(h^{2}=c^{2}=0.4\right.$, Table 2$)$. This was not the case with family selection where inbreeding was maintained at the same levels than with RM. Note that matings following sib selection were based on family means and this led to an increased probability of mating members of the same family. In contrast, matings following family selection were based on individual phenotypic values.

The effect of AM and DM on genetic gain was a consequence of the changes in the additive genetic variance. Under family selection, the variance with AM was always higher than that with RM across generations, particularly for $h^{2}=c^{2}=0.4$ (Fig. 1). Fig. 2 shows clearly that the increase in total genetic variance and therefore in genetic gain with AM was due to the increased between-family component. The effect of AM on the between-family component and on genetic gain was exacerbated by the presence of common environmental effects (Fig. 1 and Table 2). The combined effect of $\mathrm{AM}$ and $c^{2}$ produced an increase in the between-family component of the variance in the first generation of selection. The opposite occurred under DM, which led to a lower betweenfamily variance (and lower genetic gain) than RM. On the other hand, the within-family component (i.e. Mendelian segregation) was affected very little by the mating system because the inbreeding was very low under family selection when $25 / 50$ families were selected ( $F$ was about 0.09 at $t=10$ ).

Under sib selection, the total genetic variance with AM was also higher than that observed with RM in early generations (Figs. 1 and 3) due to the increased between-family component of the genetic variance (Fig. 3). However, after a number of generations (depending on $h^{2}$ and $c^{2}$ ) the between-family and total variances decreased with AM even below the value observed with RM (Fig. 1). Also, there was a drastic

Table 1

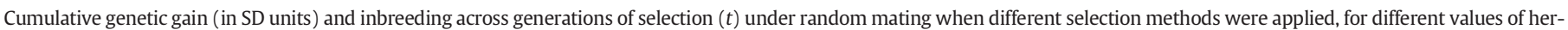
itability $\left(h^{2}\right)$ and proportion of phenotypic variance due to common environmental effects $\left(c^{2}\right)$.

\begin{tabular}{|c|c|c|c|c|c|c|c|c|c|c|c|c|c|c|c|}
\hline \multirow[t]{2}{*}{$c^{2}$} & \multirow[t]{2}{*}{$t$} & \multicolumn{7}{|c|}{ Genetic gain } & \multicolumn{7}{|c|}{ Inbreeding } \\
\hline & & Ind & $\mathrm{Fam}_{1}$ & $\mathrm{Fam}_{25}$ & $\mathrm{Sib}_{1}$ & $\mathrm{Sib}_{25}$ & Comb & With & Ind & $\mathrm{Fam}_{1}$ & $\mathrm{Fam}_{25}$ & $\mathrm{Sib}_{1}$ & $\mathrm{Sib}_{25}$ & Comb & With \\
\hline \multicolumn{16}{|c|}{$h^{2}=0.1$} \\
\hline \multirow[t]{3}{*}{0.0} & 1 & 0.24 & 0.45 & 0.16 & 0.45 & 0.16 & 0.49 & 0.11 & 0.02 & 0.38 & 0.02 & 0.38 & 0.02 & 0.24 & 0.01 \\
\hline & 5 & 1.12 & 1.48 & 0.68 & 1.48 & 0.66 & 1.69 & 0.56 & 0.06 & 0.74 & 0.05 & 0.74 & 0.05 & 0.51 & 0.02 \\
\hline & 10 & 2.11 & 2.08 & 1.30 & 2.07 & 1.27 & 2.62 & 1.11 & 0.10 & 0.91 & 0.09 & 0.91 & 0.09 & 0.71 & 0.03 \\
\hline \multirow[t]{3}{*}{0.4} & 1 & 0.24 & 0.16 & 0.03 & 0.17 & 0.05 & 0.24 & 0.14 & 0.06 & 0.38 & 0.02 & 0.38 & 0.02 & 0.08 & 0.01 \\
\hline & 5 & 1.08 & 0.44 & 0.26 & 0.42 & 0.27 & 1.06 & 0.71 & 0.18 & 0.74 & 0.05 & 0.74 & 0.05 & 0.22 & 0.02 \\
\hline & 10 & 1.94 & 0.58 & 0.52 & 0.60 & 0.54 & 1.87 & 1.45 & 0.30 & 0.91 & 0.09 & 0.91 & 0.09 & 0.38 & 0.03 \\
\hline \multicolumn{16}{|c|}{$h^{2}=0.4$} \\
\hline \multirow[t]{3}{*}{0.0} & 1 & 0.96 & 0.99 & 0.35 & 0.96 & 0.34 & 1.16 & 0.50 & 0.04 & 0.38 & 0.02 & 0.38 & 0.02 & 0.14 & 0.01 \\
\hline & 5 & 3.93 & 3.43 & 1.42 & 3.35 & 1.42 & 4.26 & 2.43 & 0.10 & 0.74 & 0.05 & 0.74 & 0.05 & 0.31 & 0.02 \\
\hline & 10 & 7.05 & 4.83 & 2.74 & 4.74 & 2.69 & 7.02 & 4.74 & 0.16 & 0.91 & 0.09 & 0.91 & 0.09 & 0.46 & 0.03 \\
\hline \multirow[t]{3}{*}{0.4} & 1 & 0.97 & 0.58 & 0.18 & 0.51 & 0.18 & 0.99 & 0.69 & 0.09 & 0.38 & 0.02 & 0.38 & 0.02 & 0.06 & 0.01 \\
\hline & 5 & 3.86 & 1.69 & 0.88 & 1.50 & 0.91 & 4.08 & 3.35 & 0.23 & 0.74 & 0.05 & 0.74 & 0.05 & 0.15 & 0.02 \\
\hline & 10 & 6.49 & 2.19 & 1.72 & 2.10 & 1.73 & 7.18 & 6.51 & 0.38 & 0.91 & 0.09 & 0.91 & 0.09 & 0.25 & 0.03 \\
\hline
\end{tabular}

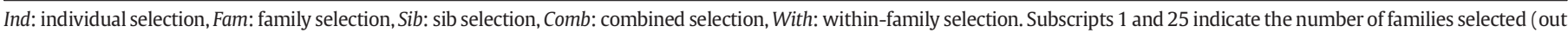
of 50 families). 
Family selection

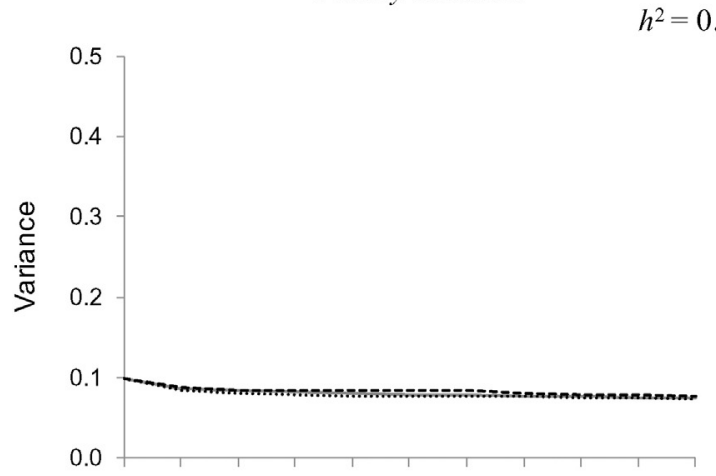

$$
c^{2}=0.0
$$
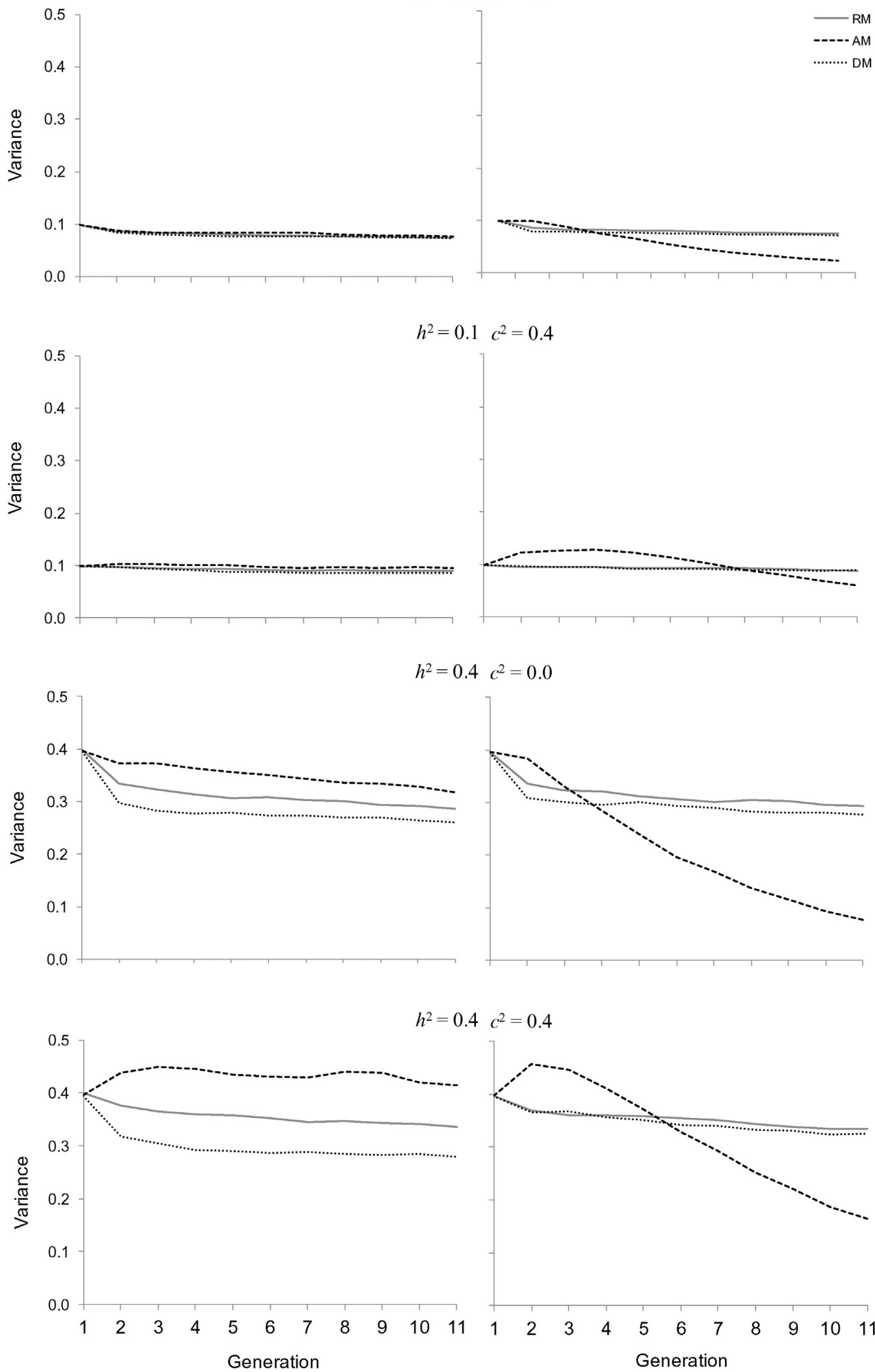

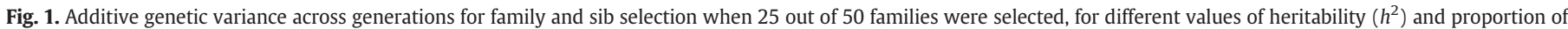
phenotypic variance due to common environmental effects $\left(c^{2}\right)$.

decrease in the within-family component, particularly for $h^{2}=0.4$. The former was a consequence of the high levels of inbreeding ( $F$ was up to 0.4 at $t=10$ ), while the decrease in the between-family variance in later generations was a consequence of the selection, this variance becoming exhausted because many loci might achieve fixation (Fig. 3). The increase in variance observed in early generations under AM 
Table 2

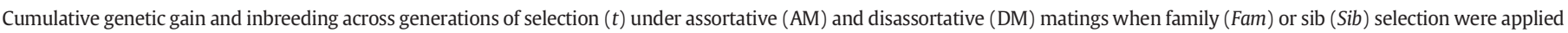

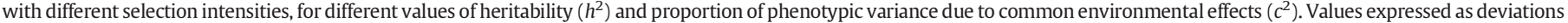
from random mating (RM).

\begin{tabular}{|c|c|c|c|c|c|c|c|c|c|c|c|c|c|}
\hline \multirow[t]{3}{*}{$c^{2}$} & \multirow[t]{3}{*}{$t$} & \multicolumn{8}{|c|}{ Genetic gain } & \multicolumn{4}{|c|}{ Inbreeding } \\
\hline & & \multicolumn{2}{|l|}{$\mathrm{Fam}_{1}$} & \multicolumn{2}{|c|}{$\mathrm{Fam}_{25}$} & \multicolumn{2}{|l|}{$\mathrm{Sib}_{1}$} & \multicolumn{2}{|l|}{$\mathrm{Sib}_{25}$} & \multicolumn{2}{|l|}{$\overline{\mathrm{Fam}_{25}}$} & \multicolumn{2}{|l|}{$\mathrm{Sib}_{25}$} \\
\hline & & AM & DM & AM & $\mathrm{DM}$ & $\mathrm{AM}$ & $\mathrm{DM}$ & AM & DM & $\mathrm{AM}$ & DM & AM & DM \\
\hline \multicolumn{14}{|c|}{$h^{2}=0.1$} \\
\hline \multirow[t]{6}{*}{0.0} & 1 & -0.01 & -0.01 & 0.00 & 0.00 & -0.01 & -0.02 & 0.00 & 0.01 & 0.000 & 0.000 & 0.003 & -0.003 \\
\hline & 2 & 0.02 & 0.02 & 0.00 & -0.01 & -0.01 & -0.02 & 0.03 & 0.00 & 0.000 & 0.000 & 0.010 & -0.011 \\
\hline & 3 & 0.02 & 0.01 & 0.00 & -0.02 & 0.00 & 0.00 & 0.06 & -0.01 & 0.000 & 0.000 & 0.023 & -0.024 \\
\hline & 4 & 0.02 & 0.00 & 0.00 & -0.02 & 0.02 & 0.02 & 0.09 & -0.02 & 0.000 & 0.000 & 0.044 & -0.045 \\
\hline & 5 & 0.03 & 0.02 & 0.01 & -0.03 & 0.00 & 0.00 & 0.10 & -0.03 & 0.000 & 0.000 & 0.070 & -0.072 \\
\hline & 10 & 0.00 & -0.04 & 0.05 & -0.06 & -0.07 & -0.05 & 0.04 & -0.08 & 0.001 & -0.002 & 0.311 & -0.313 \\
\hline \multirow[t]{6}{*}{0.4} & 1 & -0.02 & -0.01 & 0.03 & 0.02 & -0.01 & 0.01 & 0.02 & 0.02 & 0.000 & 0.000 & 0.003 & -0.002 \\
\hline & 2 & 0.02 & 0.02 & 0.02 & 0.02 & -0.04 & -0.01 & 0.02 & 0.00 & 0.000 & 0.000 & 0.008 & -0.008 \\
\hline & 3 & 0.01 & 0.01 & 0.01 & 0.00 & -0.02 & -0.03 & 0.04 & -0.01 & 0.000 & 0.000 & 0.016 & -0.016 \\
\hline & 4 & -0.01 & -0.03 & 0.01 & -0.01 & 0.03 & 0.03 & 0.13 & 0.03 & 0.001 & -0.001 & 0.030 & -0.030 \\
\hline & 5 & 0.01 & -0.01 & 0.02 & -0.01 & 0.01 & 0.02 & 0.17 & 0.00 & 0.000 & -0.001 & 0.050 & -0.049 \\
\hline & 10 & 0.01 & -0.03 & 0.05 & -0.05 & -0.02 & -0.01 & 0.32 & -0.03 & 0.001 & -0.003 & 0.202 & -0.201 \\
\hline \multicolumn{14}{|c|}{$h^{2}=0.4$} \\
\hline \multirow[t]{6}{*}{0.0} & 1 & -0.03 & -0.03 & 0.00 & 0.00 & 0.00 & -0.02 & 0.00 & 0.01 & 0.000 & 0.000 & 0.003 & -0.003 \\
\hline & 2 & 0.04 & -0.14 & 0.04 & -0.05 & -0.02 & -0.05 & 0.04 & -0.01 & 0.001 & -0.001 & 0.010 & -0.011 \\
\hline & 3 & 0.12 & -0.21 & 0.09 & -0.10 & 0.01 & -0.01 & 0.10 & -0.04 & 0.002 & -0.002 & 0.023 & -0.025 \\
\hline & 4 & 0.12 & -0.27 & 0.13 & -0.15 & 0.05 & 0.01 & 0.14 & -0.05 & 0.002 & -0.003 & 0.044 & -0.047 \\
\hline & 5 & 0.11 & -0.30 & 0.20 & -0.18 & 0.03 & -0.04 & 0.16 & -0.07 & 0.004 & -0.005 & 0.069 & -0.072 \\
\hline & 10 & 0.12 & -0.25 & 0.43 & -0.37 & -0.03 & 0.00 & -0.01 & -0.12 & 0.008 & -0.011 & 0.288 & -0.293 \\
\hline \multirow[t]{6}{*}{0.4} & 1 & -0.01 & -0.04 & 0.02 & 0.02 & 0.05 & 0.02 & 0.02 & 0.03 & 0.000 & 0.000 & 0.003 & -0.003 \\
\hline & 2 & 0.15 & -0.11 & 0.07 & -0.03 & 0.03 & -0.02 & 0.08 & -0.01 & 0.001 & -0.001 & 0.010 & -0.009 \\
\hline & 3 & 0.24 & -0.32 & 0.14 & -0.09 & 0.03 & -0.03 & 0.17 & -0.03 & 0.002 & -0.003 & 0.022 & -0.021 \\
\hline & 4 & 0.29 & -0.43 & 0.21 & -0.17 & 0.08 & -0.01 & 0.30 & 0.00 & 0.004 & -0.005 & 0.039 & -0.038 \\
\hline & 5 & 0.30 & -0.51 & 0.29 & -0.23 & 0.10 & 0.05 & 0.36 & -0.03 & 0.005 & -0.006 & 0.066 & -0.064 \\
\hline & 10 & 0.43 & -0.59 & 0.66 & -0.52 & 0.13 & -0.01 & 0.54 & -0.09 & 0.013 & -0.015 & 0.275 & -0.271 \\
\hline
\end{tabular}

Fam: family selection, Sib: sib selection. Subscripts 1 and 25 indicate the number of families selected (out of 50 families).

when common environmental effects were present was higher with sib than with family selection because in the former case matings were based on family means. The higher probability of mating individuals within families under sib selection also explains the higher advantage of AM in terms of genetic gain when compared to RM when $c^{2}=0.4$ in early generations (Table 1 ).

\section{Discussion}

In this simulation study, the effect of the mating scheme on genetic gain and inbreeding has been explored for aquaculture selection programs where tank effects and large family sizes are common. To our knowledge, this is the first study where the effect of the mating scheme is explored in an aquaculture context under different selection methods (Falconer and Mackay 1996). Our results suggest that under particular selection methods (family and sib selection), the response to selection was increased when AM was implemented instead of RM. The advantage of AM increased when common environmental effects were present. Contrarily, decreased genetic gain was observed when DM was implemented, except for the case of within-family selection.

With the exception of some particular scenarios under individual and combined selection that showed a small advantage of AM over RM in later generations, the only selection methods that showed a clear effect of AM on genetic gain were family and sib selection. Several theoretical studies (e.g. Fisher, 1918; Wright, 1921; Baker, 1973; Lande, 1977; Rosvall and Mullin, 2003) have investigated the effects of AM under polygenic inheritance and have predicted that this mating system increase the genetic variance as a result of genetic covariations among loci. Empirical studies have also showed higher responses to selection with AM than with RM, although the differences were not always significant (McBride and Robertson, 1963; Wilson and Kyle, 1965; Mwenya et al., 1984; Campo and García-Gil, 1994). Here, the large family sizes simulated represented an optimal condition for family (and sib) selection methods (Falconer and Mackay, 1996). Under a low selection intensity (i.e. selecting 25 out of 50 families), family and sib selection lead to a high increase in the between-family component of the genetic variance, which is the component affected by AM. In addition, the presence of common environmental effects favored family selection over other methods and reinforced the advantage of AM over RM. All of this explains the clear effect of AM on genetic gain under family and sib selection compared to RM. In particular, genetic gain increased up to $80 \%$ at $t=1$ and $40 \%$ at $t=10$ (for different values of $h^{2}$ and $c^{2}=0.4$ ).

In general, traditional selection methods giving higher genetic gains also give higher inbreeding (Belonski and Kennedy, 1988; Villanueva et al., 1995). Here, for those selection methods where AM had a higher effect on genetic gain (i.e. family and sib selection when selecting 25 out of 50 families) only family selection kept inbreeding at low levels $(F<0.1)$ after 10 generations. However, with sib selection, inbreeding increased by $300 \%$ ( $F$ ranged from 0.3 to 0.4 , Table 2 ) when compared with that obtained under RM (0.09). The difference in inbreeding levels between both methods can be explained by the fact that matings were based on individual values following family selection but on family means following sib selection as in this latter case individual values are unavailable in practice.

It is interesting to note that the advantage of AM over RM was higher when common environmental (tank) effects were present. In the presence of common environmental effects the between-family component of the additive genetic variance is increased, because phenotypes are differentially affected by the environment within each tank. This translates in an increased additive variance due to the joint effect of AM and $c^{2}$, which can be used by selection in the following generation. The advantage of AM over RM is maintained across generations. On the other hand, the within-family component of the variance is only affected by inbreeding and not by the mating scheme. In situations where nested designs are used (e.g. one male mated to several females), the rate of inbreeding and the rate of response would increase when compared to single pair matings (the scenario simulated here), as, for the same number of dams, the number of sires decreases. In that case, the effect of AM 
$V_{A B}$

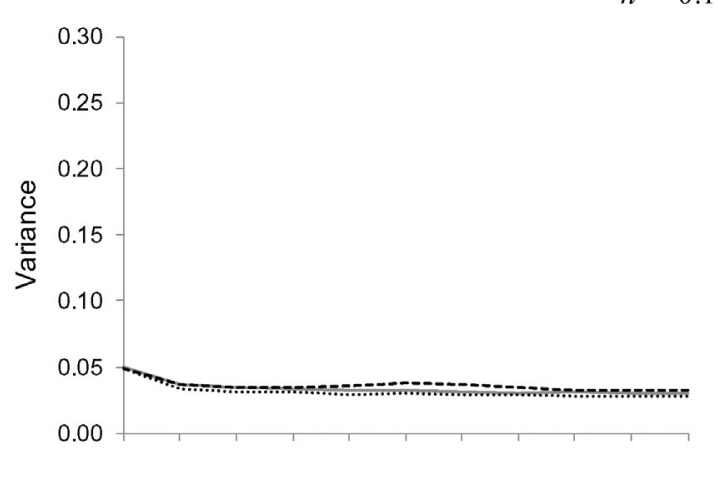

$h^{2}=0.1 \quad c^{2}=0.4$

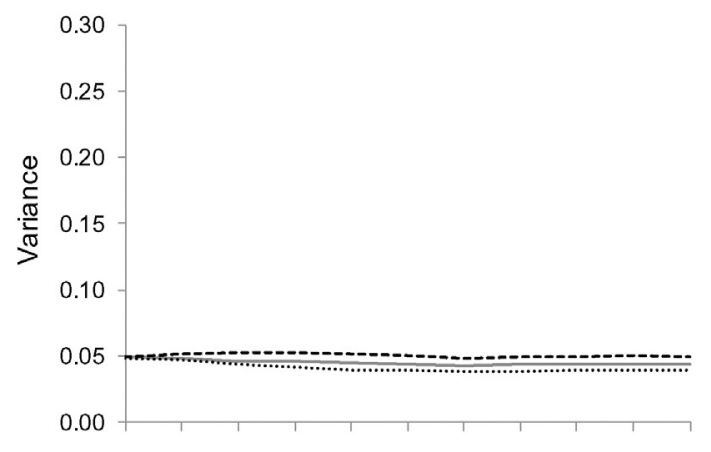

$h^{2}=0.4 \quad c^{2}=0.0$
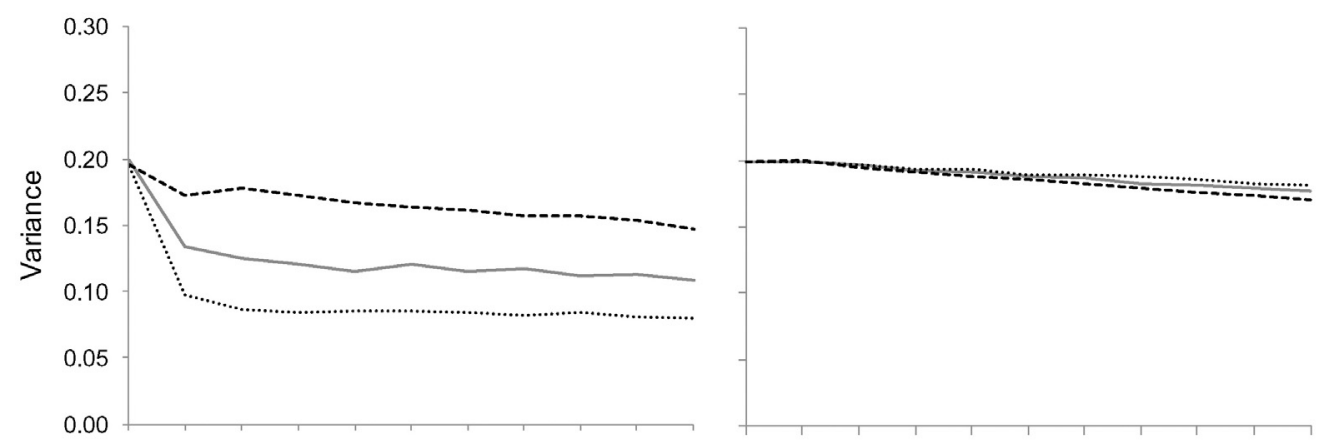

$$
h^{2}=0.4 \quad c^{2}=0.4
$$
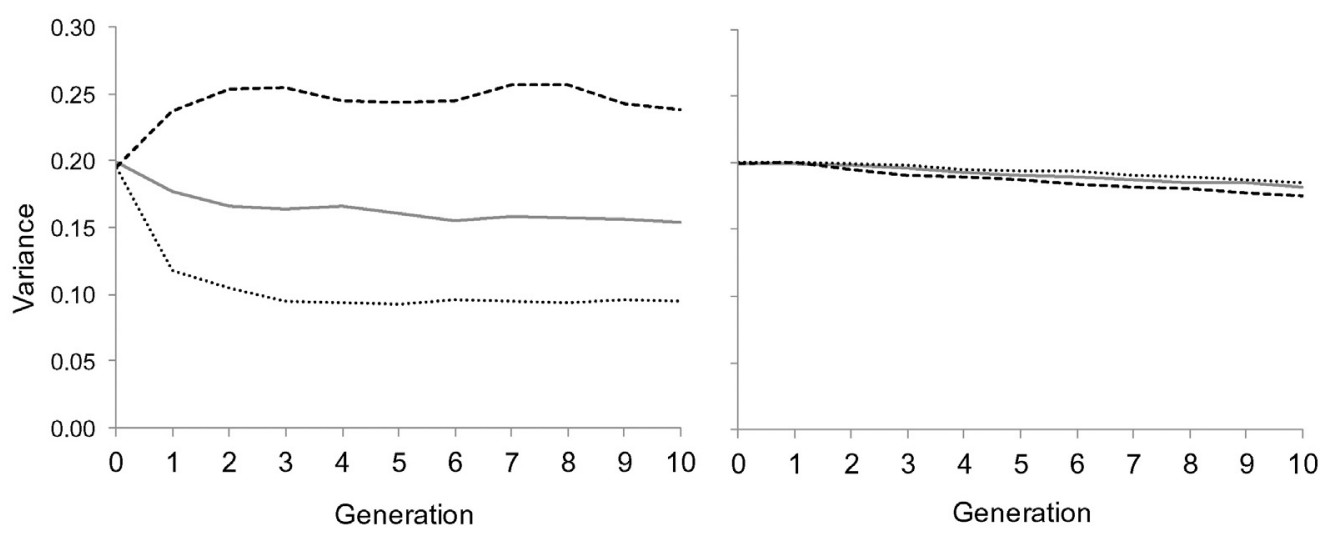

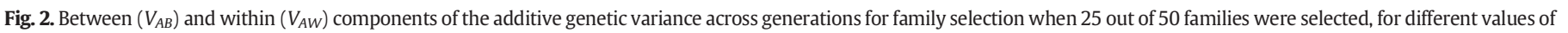
heritability $\left(h^{2}\right)$ and proportion of phenotypic variance due to common environmental effects $\left(c^{2}\right)$.

would be reduced because of the higher the selection intensity, the lower the between-family variance and the lower the correlation between parents.
The impossibility of tagging newborn fish physically leads to the general practice of rearing families in separate tanks until the individuals are large enough to be individually tagged. Under this particular 

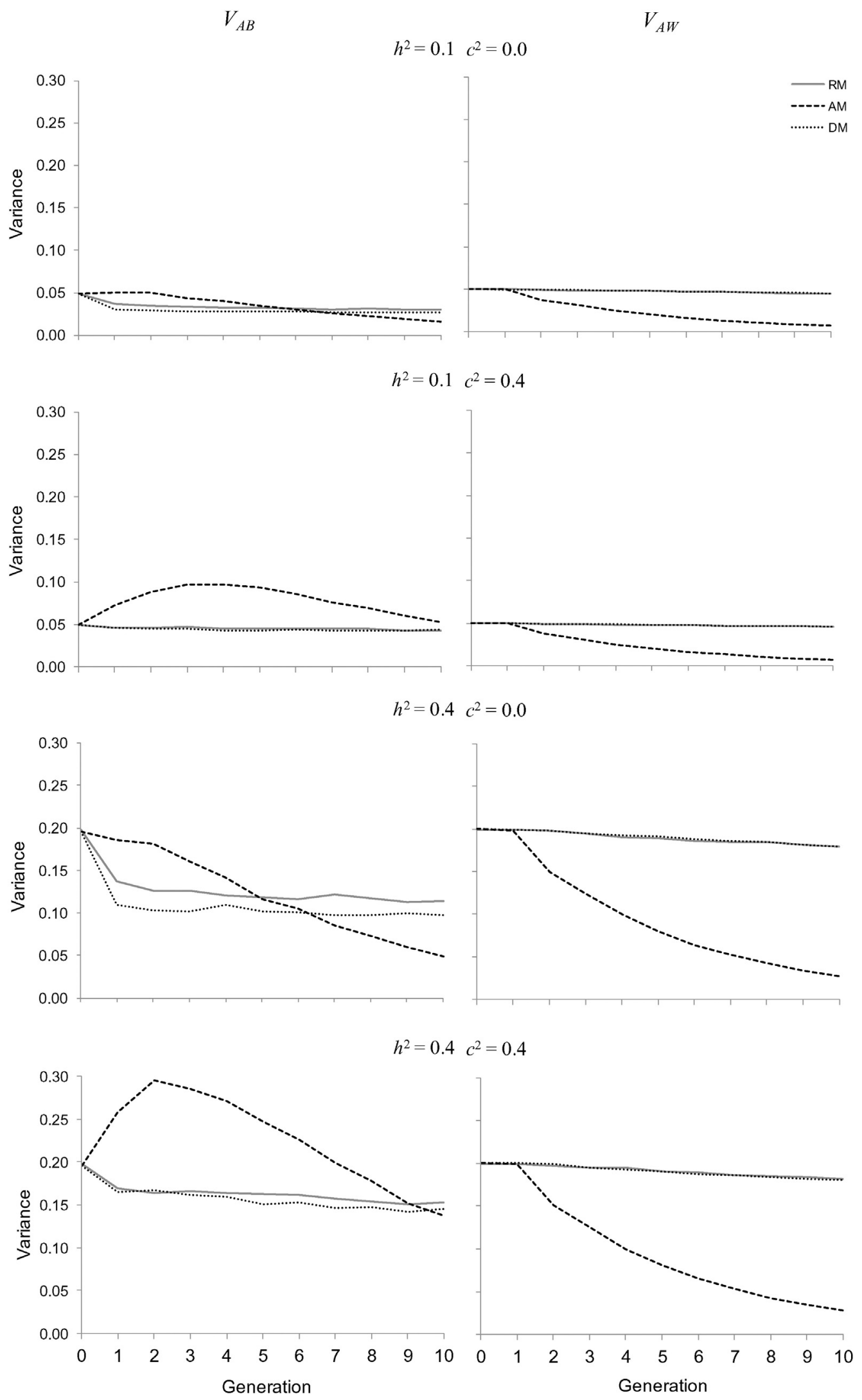

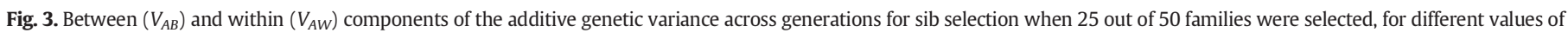
heritability $\left(h^{2}\right)$ and proportion of phenotypic variance due to common environmental effects $\left(c^{2}\right)$.

context, members of a full sib family share a common environmental during the early rearing stage and this may imply an environmental correlation among them. Previous studies have reported relatively large estimates of common environmental effects in fish. Winkelman and Peterson (1994) obtained values up to $28 \%$ of the total phenotypic variance for body weight in chinook salmon. Rye and Mao (1998) obtained 
estimates of common environmental effects between 4 and $6 \%$ for body weight in Atlantic salmon, while Gallardo et al. (2010) reported significant but low ( 0.7 to $6 \%$ of the total phenotypic variance) common environmental effects in coho salmon selected for the same trait. It is therefore important to take into account common environmental effects when investigating the effect of the mating scheme on genetic gain, particularly in aquaculture breeding programs.

\section{Conclusions}

To our knowledge, this is the first study that investigates the effect of the mating scheme on genetic gain and inbreeding in aquaculture breeding programs. Here we have shown that AM in an aquaculture context where family sizes are large and tank effects are present, can substantially enhance the response to selection, particularly when family selection methods are applied. Our results suggest that AM in the presence of common environmental variance may be considered in selection programs in aquaculture.

\section{Acknowledgments}

The research leading to these results has received funding from the European Union's Seventh Framework Programme (KBBE.2013.1.210) under grant agreement $n^{\circ} 613611$.

\section{References}

Baker, R.J., 1973. Assortative mating and artificial selection. Heredity 31, 231-238. Belonski, G.M., Kennedy, B.W., 1988. Selection o individual phenotype and best linear unbiased predictor of breeding value in a closed swine herd. J. Anim. Sci. 66, 1124-1131. Breese, E.L., 1956. The genetical consequences of assortative mating. Heredity 10, 32-343. Bulmer, M.G., 1971. The effect of selection on genetic variability: a simulation study. Genet. Res. 28, 101-117.
Campo, J.L., García-Gil, M., 1994. J. Anim. Breed. Genet. 111, 213-219.

Falconer, D.S., Mackay, T.F.C., 1996. Introduction to Quantitative Genetics. fourth ed. Longman, London and New York.

Fisher, R.A., 1918. The correlation between relatives on the supposition of Mendelian inheritance. Trans. R. Soc. Edinb. Earth Sci. 52, 399-433.

Gallardo, J.A., Lhorente, J.P., Neira, R., 2010. The consequences of including non-additive effects on the genetic evaluation of harvest body weight in Coho salmon (Orcorhynchus kisutch). Genet. Sel. Evol. 42, 19.

Gjedrem, T., 2012. The importance of selective breeding in aquaculture to meet future demands for animal protein: A review. Aquaculture 350-353, 117-129.

Gjedrem, T., Baranski, M., 2009. In: J.N., N. (Ed.), Selective Breeding in Aquaculture: An Introduction. Springer, Dordrecht Heidelberg London New York.

James, J.W., McBride, G., 1958. The spread of genes by natural and artificial selection in a closed poultry flock. J. Genet. 56, 55-62.

Lande, R., 1977. The influence of the mating system on the maintenance of genetic variability in polygenic characters. Genetics $86,485-498$.

Lynch, M., Walsh, B., 1998. Genetics and Analysis of Quantitative Traits. Sinauer Associates, Sunderland, MA.

McBride, G., Robertson, A., 1963. Selection using assortative mating in Drosophila melanogaster. Genet. Res. 4, 356-369.

Mwenya, W.N.M., Gianola, D., Baker, R.L., 1984. Assortative mating and selection: an experimental studio with Tribolium castaenum. Proc. 34th A. Meet. Eur. Ass. Anim. Prod., Anim. Genetics Commn., Madrid 1, 118-119.

Neira, R., Díaz, N.F., Gall, G.A.E., Gallardo, J.A., Lhorente, J.P., Manterola, R., 2006. Genetic improvement in Coho salmon (Oncorhynchus kitsutch). I: Selection response and inbreeding depression on harvest weight. Aquaculture 257, 1-4.

Rosvall, O., Mullin, T.J., 2003. Positive assortative mating with selection restrictions on group coancestry enhances gain while conserving genetic diversity in long-term forest tree breeding. Theor. Appl. Genet. 107, 629-642.

Rye, M., Mao, I.L., 1998. Nonadditive genetic effects and inbreeding depression for body weight in Atlantic salmon (Salmo salar L.). Livest. Prod. Sci. 57, 15-22.

Villanueva, B., Woolliams, J.A., Simm, G., 1995. The effect of improved reproductive performance on genetic gain and inbreeding in MOET breeding schemes for beef cattle. Genet. Sel. Evol. 27, 347-363.

Wilson, S.P., Kyle, W.H., 1965. The effects of mating systems and selection on pupa weight in Tribolium. Genet. Res. Camb. 6, 341-351.

Winkelman, A.M., Peterson, R.G., 1994. Heritabilities, dominance variation, common environmental effects and genotype by environmental interactions for weight and length in chinook salmon. Aquaculture 125, 17-30.

Wright, S., 1921. Systems of mating. Genetics 6, 111-178. 